\title{
ARQUITECTURA DE LA COMPLEJIDAD Y COMPLEJIDAD DE LA ARQUITECTURA
}

\section{Sergio PORTEL}

Arquitecto, profesor adjunto, Arquitectura III. UPB

Palabras Clave: Complejidad, arquitectura, currículo.

Keywords: Complexity, architecture, curriculum.

\section{RESUMEN}

Andamos transitando un camino dilemático, queremos enseñar la complejidad de la realidad, pero sin embargo la diseccionamos y la recortamos de tal manera que solo encontramos fragmentos, rastros parciales de ella, mutilando nuestra posibilidad de comprenderla. En cambio, si pretendemos mantener la riqueza de elementos y relaciones desde un enfoque complejo, nos encontramos en dificultades, sin las herramientas conceptuales e instrumentales para abordarla. El paradigma de la simplicidad, de la especialidad, de la hegemonía de las disciplinas que saben mucho de poco, está en crisis, incluso o en mayor medida en nuestras aulas universitarias. Animamos a discutir desde nuestras prácticas los alcances o repercusiones de este paradigma.

\section{ABSTRACT}

We walk travelling a dilemmatic way, we want to teach the complexity of the reality, but nevertheless we cut and dissected it in such a way that only we find fragments, partial tracks of her, mutilating our possibility of understanding it. However, if we try to support the wealth of elements and relations from a complex approach without the conceptual and instrumental tools, we are in difficulties. The paradigm of the simplicity, of the speciality, of the hegemony of the disciplines that knows a lot of of little, is in crisis, even in major measure in our university classrooms. We encourage to discuss from our practices the scopes or repercussions of this paradigm. 


\section{OBJETIVOS}

El objetivo del trabajo está asociado a una serie de discusiones acerca del actual plan de estudios de la carrera de Arquitectura de la FAU, UNNE, y pretende poner de relieve las limitaciones de los marcos teórico-conceptuales que configuran y naturalizan determinadas prácticas docentes.

\section{INTRODUCCIÓN}

En el ámbito académico escuchamos con frecuencia utilizar el término complejidad con una multiplicidad de acepciones que contribuyen en mínimo grado a su comprensión. Es así que el término complejo puede ser utilizado como sinónimo de complicado o difícil. Calificamos como complejo algún hecho en el que confluyen múltiples aspectos, o simplemente algo que representa algún tipo de dificultad para su cabal comprensión. El concepto de complejidad ha ido inundando diferentes disciplinas y con ello resignificándose, pero esa diversificación semántica trae confusión y equívocos aparejados, sobre todo cuando no se toman adecuados recaudos. Además, hay una suerte de moda o pose en la que hablar de la complejidad nos incluye en los selectos circulos de la vanguardia académica. Dice CARLOS REYNOSO "Un buen número de sociólogos y antropólogos piensa que con hacer referencia ocasional al pensamiento de Morin, yuxtaponer enfoques como él lo hace o agendar objetivos morinianos a tener en cuenta, alcanza para situar un desarrollo teórico, cualquiera sea su objeto, en un plano de complejidad". ${ }^{1}$

Este artículo pretende contribuir al debate en el campo de la enseñanza de la arquitectura acerca de los obstáculos que implica la fragmentación de los contenidos en el proceso de comprensión de los problemas.

\section{COMPLICACIONES DE LA COMPLEJIDAD}

Lo que le ocurre al concepto de la complejidad ya le ocurrió hace algunos años al concepto de paradigma, que terminó siendo una suerte de comodín de uso obligado para todo aquello que implicara un cambio importante. En nuestras escuelas de arquitectura, los términos complejo y complejidad son utilizados en diferentes contextos y con diferentes acepciones.

Esto que pasa en nuestra doméstica vida académica no constituye más que una réplica de lo que puede apreciarse en otros ámbitos de discusión teórica mundial. Tomemos a modo de ejemplo tres autores de relevancia mundial.

HERBERT SIMON, Premio Nobel de Economía, en su ensayo "La arquitectura de la complejidad", en el que trata diversos aspectos relacionados con la complejidad de los sistemas, propone una de las parábolas emblemáticas del estudio de la complejidad, que ilustra perfectamente cómo se puede tratar esta y los problemas derivados de ella. El cuento, o la parábola, como lo llama SIMON, dice algo más o menos así: Hubo una vez dos relojeros, llamados Tempus y Hora, que fabricaban relojes de excelente calidad. Los dos gozaban de una gran reputación y tenían un gran número de clientes. Sin embargo, Hora prosperó, mientras que Tempus se fue empobreciendo cada vez más hasta que finalmente perdió el negocio. La razón de ello requiere una explicación previa. Los relojes que ambos hacían estaban compuestos por unas 1000 piezas. Tempus ensamblaba los relojes de tal manera que si interrumpía el proceso de fabricación en cualquier punto que no fuera el final de la construcción del reloj, este se desmontaba y había que empezar el proceso de nuevo desde el principio. A medida que tenía más clientes que llegaban a su taller a formalizar sus encargos, sufría más interrupciones y se le hacía más difícil tener tiempo suficiente para acabar un reloj. 
la complejidad se impone de entrada como imposibilidad de simplificar; ella surge alli donde la unidad compleja produce sus emergencias..."

Los relojes que hacía Hora no eran menos complejos, pero estaban diseñados de tal forma que se podían construir partes completas de manera independiente, cada una de ellas de unas diez piezas. Cada parte de estas servía para construir otra subparte más grande de unas cien piezas, y diez de estas subpartes formaban el reloj completo. De aquí que, cuando Hora se veía interrumpido por un cliente, con la misma frecuencia que Tempus, el trabajo que perdía era solo una pequeña parte del trabajo total, consiguiendo montar un reloj en mucho menos tiempo que Tempus.

La moraleja evidente de esta parábola es que la complejidad se puede comprender y manejar más eficientemente por medio de módulos o jerarquías, simplificando el problema pero sin distorsionar su naturaleza.

EDGAR MORÍN, uno de lo filósofos que más ha trabajado la complejidad, se refiere a ella en estos términos: "la complejidad se impone de entrada como imposibilidad de simplificar; ella surge alli donde la unidad compleja produce sus emergencias, alli donde se pierden las distinciones y claridades de las identidades y las causalidades, allí donde los desórdenes y las incertidumbres perturban los fenómenos, alli donde el sujeto-observador sorprende su propio rostro en el objeto de observación, allí donde las antinomias hacen divagar el curso del razonamiento".

Nuestro ROLANDO GARCÍA² sugiere ver la noción de sistema complejo como "una representación de un recorte de la realidad compleja, conceptualizado como una totalidad organizada, de ahí la denominación de sistema en la cual los elementos no son 'separables' y por lo tanto no pueden ser estudiados aisladamente; los elementos de un sistema complejo son 'interdefinibles'".

Explica GARCÍA: "Los sistemas complejos están constituidos por elementos heterogéneos en interacción y de allí su denominación de complejos lo cual significa que sus subsistemas pertenecen a los 'dominios materiales' de muy diversas

[2 ] ROLANDO GARCÍA. "Sistemas Complejos" (2007). disciplinas", y en otro punto señala: "la complejidad está asociada con la imposibilidad de considerar aspectos particulares de un fenómeno, proceso o situación a partir de una disciplina específica".

\section{COMPLEJIDAD Y REALIDAD}

El mundo que se nos presenta es complejo, y el observador simplemente copia esa complejidad para poder entenderlo según la ilusión empirista, ¿o es un recurso de la mente para poder ordenar el caos con el que se nos presenta ese mundo? Volvemos a la vieja discusión epistemológica de la verdad: ¿la complejidad está en el objeto, o es un dispositivo que está en el observador para poder inteligir la realidad? O quizá lo que consideramos "lo real" es el resultado de un proceso histórico social de asimilación-acomodación-equilibración de un mundo que construimos y a la vez nos construye.

Definimos nuestros objetos de estudios según los conceptos y las herramientas de las disciplinas, y las disciplinas se van configurando según los objetos que estudiamos. Dice GARCíA que la complejidad está asociada con la imposibilidad de considerar aspectos particulares de un fenómeno, proceso o situación a partir de una mono-disciplina.

Si coincidimos con este postulado, la idea de complejidad adquiere en este enfoque otra dimensión, referida al modo de abordaje científico tal como lo planteóPIAGETparadefinirlosdominiosdelaciencia: a) Dominio material : el conjunto de objetos a los cuales se refiere una disciplina.

b) Dominio conceptual: el conjunto de teorías o conocimientos sistematizados elaborados por cada ciencia acerca de su dominio material.

c) Dominio epistemológico interno: corresponde al análisis de los fundamentos de cada disciplina, es decir, a la crítica de las teorías de su dominio conceptual. 
d) Dominio epistemológico derivado: analiza las relaciones entre el sujeto y el objeto de conocimiento, el marco más general, comparando los resultados de una disciplina con los de otras.

Esto nos lleva a reflexionar acerca de los campos de actuación del arquitecto en la realidad. Por ejemplo, ¿se puede sostener la idea de que el urbanismo es una disciplina dominada por los arquitectos? Y paralelamente, si recortamos una porción de ciudad, vemos que allí se desarrolla un complejo entramado de actividades, de actores económicos, culturales, sociales, productivos, tecnológicos, políticos etc. ¿Dónde se estudia interdisciplina o metodologías interdisciplinarias? ¿Cómo aprendemos a dialogar con economistas, geógrafos, sociólogos y otros que saben de otras porciones del mundo de lo real?

\section{Complejidad de la arquitectura}

Si las prácticas interdisciplinarias están ausentes en la academia, ¿cómo haremos para que nuestras visiones del campo de lo arquitectónico no sean meras construcciones imaginarias en lugar de modelos capaces de interpelar la realidad?

Vamos a husmear un poco acerca de qué se dice de la complejidad en los corrillos de arquitectos dedicados a la docencia y algunos otros que escribieron sobre la temática, a fin de dilucidar los laberintos de nuestro peculiar objeto de estudio.

En el ámbito de los talleres se acuerda a menudo que un objeto arquitectónico es más complejo que otro en la medida en que contenga mayor cantidad de elementos o que las relaciones sean de tal magnitud que se torne dificil comprenderlas. Por ejemplo, un hospital sería más complejo que un galpón de depósito, o un hospital regional sería más complejo que un hospital de una localidad pequeña. En cambio, en la jerga de la salud se denominará de alta complejidad según la capacidad tecnológica y de recursos humanos del centro de realizar intervenciones que requieren un alto grado de especialización.

Pero también podemos escuchar que la complejidad está dada por una cuestión metodológica $u$ operativa, entonces una tienda de emergencia o catástrofes puede ser más compleja que una torre de viviendas. Se confunde a menudo una cuestión de escala, magnitud física con cantidad de componentes o relaciones.

En la historia la noción de complejidad también estuvo sometida a difersas interpretaciones. Los principales exponentes de la arquitectura moderna abogaron en cierta medida por la simplificación del mundo a partir de un saber tecno-científico. El menos es más de MIES, llevado al paroxismo en la Farnsworth House, resultó ser que algunos casos menos simplemente eran menos, ignorando la forma de vida del usuario: paños vidriados que en invierno producían una desagradable condensación, mala resolución hidráulica de la cubierta, efecto invernadero en verano, escasa empatía de la propuesta con la comitente, etc. La simplificación artificial de un problema parece ser que no arriba necesariamente a una solución, sobre todo si la simplificación se impone al problema provocando contradicciones profundas entre la demanda y la intervención técnica.

LUDOVICO QUARONI ${ }^{3}$ propone: "Es posible considerar esta estructura edificio como una entidad autónoma de dependencias internas, es decir, como una estructura que comprende al mismo tiempo forma y contenidos, como las dos páginas de un mismo folio, folio que tiene su propia estructura física y luego tecnológica, la una sostén de la otra y cada una la otra cosa. Es necesario hacer otra observación: que si bien las partes están subordinadas, es divisible aunque sea a través de los artificios de la abstracción analítica en varias

[3 ] Ludovico Quaroni. Proyectar un edificio (1977). 
"Las combinaciones iguales de las partes consiguen un conjunto por la superposición y la simetría y no por la dominancia y la jerarquía".

estructuras, estructura de espacios, estructura figurativa, estructura tecnológica. Si bien el modelo que sugiere pretende una mirada estructural o jerárquica, siempre queda acotada o circunscripta al edificio, al objeto material".

En Complejidad y contradicción en la arquitectura, de 1966, ROBERT VENTURI defiende una posición contraria a la arquitectura moderna, contra su pretención de buscar solo la diferencia y la novedad. Para él la complejidad de la forma arquitectónica no puede ser reducida a un solo sistema lógico y estético.

Un aceptar las complicaciones del hombre común en vez de ignorarlas y plantear un empezar de cero como hacían sus predecesores. De todas maneras, a pesar de que conocía a H. SIMÓN, ya que lo menciona en la primera parte de su libro, creo que aporta más a lo que él considera contradicciones de la arquitectura moderna con su tiempo y mucho menos en lo que respecta a la complejidad, ya que esta apunta más a una visión gestáltica de relación entre la parte y el todo.

Escribe VENTURI: "Pero una relación jerárquica más ambigua de las partes no inflexionadas crea una percepción más difícil del conjunto. Tal conjunto está compuesto de combinaciones de partes iguales. Mientras que la idea de combinaciones iguales está relacionada con el fenómeno 'ambos- $y$ ', y muchos ejemplos recurren a ambas ideas, 'ambos-y' se refiere más específicamente a la contradicción en arquitectura, mientras que las combinaciones iguales se refieren más a la unidad. Con combinaciones iguales el conjunto no depende de la inflexión, o de las relaciones más fáciles del nexo dominante, o de la regularidad generada. Por ejemplo, en la Porta Pia $(110,111)$, el número de cada tipo de elementos en la composición de la puerta y el muro, es casi igual —no domina ningún elemento-. Al ser casi iguales, las diversas formas (rectangulares, cuadradas, triangulares, segmentales y redondas) se excluye también el predominio de cualquier forma y las diversas direcciones (verticales, horizontales, diagonales y curvas), al ser muy parecidas, tienen el mismo efecto. De manera similar hay una diversidad parecida en el tamaño de los elementos. Las combinaciones iguales de las partes consiguen un conjunto por la superposición y la simetría y no por la dominancia y la jerarquía".
Algunos estilos o formatos de disciplinas, como algunas "Historias", han contribuido a la fragmentación del fenómeno arquitectónico, escencialmente al escindir el edificio del uso y del proceso de producción en el que se gestó, y en algunos casos hasta el entorno socioeconómico en el que esa obra tuvo sentido. ¿Alguien sabe cuánto costaron obras emblemáticas como el Coliseo, o Santa Sofía, cómo se organizó su materialización, cuáles fueron los recursos humanos utilizados, los problemas funcionales que tenían o sus patologías constructivas?

Los propios talleres de arquitectura se concentran en los aspectos meramente proyectuales escindidos de las lógicas productivas, de las problemáticas del mercado.

El cliente es una figura caricaturizada que siempre acepta lo que proponemos, y que está dispuesto en buen grado a financiar nuestras ocurrencias.

La materialidad es reducida a una suerte de catálogos de detalles, desconociendo el proceso en el que se desarrollan. Una carpintería es un dibujo y no un instrumento de decisión dentro de un proceso constructivo en el que intervienen diferentes actores, insumos, equipos y materiales.

La mayor renuncia a la complejidad probablemente sea la escisión entre edificio y usuario. En los talleres de arquitectura, quizá como un resabio de los postulados modernos, el usuario queda reducido a estándares, que generalmente reponden a parámetros económicos y pocas veces a la observación rigurosa de la vida de los destinatarios de nuestra producción material. Vemos y aceptamos como válidas propuestas de viviendas en las que no existen espacios ni lugares para bicicletas, juguetes, cunas, huéspedes, trastos viejos, herramientas, viejos, obesos, discapacitados, lugar de planchado, etc.

En el campo de la arquitectura, las sub-disciplinas se estudian en primer lugar de manera abstracta, 
matriz formativa que heredamos y que reproducimos, nos queda repensar rigurosamente cuáles son los aspectos que consideramos positivos y conviene mantener..."

desvinculadas de sus contextos de aplicación, y después se pretende que se resuelvan eficientemente en sus contextos. Esa fragmentación disciplinar es sumamente contraproducente para entender la inescindible convivencia en el campo de la producción del hábitat. Por ejemplo, en un edificio de departamentos producido como inversión inmobiliaria bajo la lógica del mercado, será viable, lógico y tendrá un valor formativo en la medida en que se comprenda la integralidad de ese proceso de producción del espacio. Comprender que se debe respetar un plan de negocio, que deben contemplar valores de mercado, que el programa surge de la discusión entre diferentes actores, etc.

Frente a este panorama, a esta tipología de formación, a esta matriz formativa que heredamos y que reproducimos, nos queda repensar rigurosamente cuáles son los aspectos que consideramos positivos y conviene mantener, y por el contrario, cuáles son los que deberíamos cambiar para dar cuenta de lo que consideramos un cambio de paradigma.

Podríamos convenir que debemos propiciar un modelo de aprendizaje en el que se pueda garantizar trabajar desde la complejidad de la arquitectura. Esto se logra configurando un modelo que integre, que no fragmente, que pueda comprender lo que se incribe, la decisión de realizar una obra, los procesos de definición del programa, los procesos proyectuales y los de materialización, todos ellos contextualizados en su lugar y en su tiempo.

La otra cuestión que distorsiona la comprensión del trabajo en su complejidad es creer que el alumno debe saber todo y de todo. Habitualmente no se anima al estudiante a consultar a especialistas en los diferentes subsitemas, a consultar la viabilidad de la propuesta con constructores, herreros, instaladores etc. Quizá podamos afirmar que el pensamiento complejo sea menos complicado que el pensamiento fragmentado. Si bien la idea de complejidad aplicada puede parecer a priori más difícil, resulta mucho más amigable para el estudiante porque para él tiene sentido. La especialidad, el detalle, la comprensión de la parte surge en los pliegues, en el entramado de la comprensión del problema integral.

\section{PENSAMIENTO COMPLEJO Y CURRÍCULO}

Trataremos de ir completando la idea que propone el título del artículo vinculando la arquitectura de la complejidad, su estructura conceptual y los beneficios de entender la arquitectura desde una epistemología de la complejidad.

Si entendemos entonces que la idea de complejidad no es una mera copia de las estructuras del objeto, como así tampoco un mero artificio mental para inteligir el mundo, sino el resultado de sucesivas abstraciones reflexivas en el devenir históricosocial del conocimiento de la realidad, podemos afirmar que debemos munirnos de los instrumentos apropiados para comprender e intervenir en nuestras prácticas académicas.

La utopía de explicar el mundo a través de unas pocas leyes dominó la producción científica del siglo XIX y parte del XX. El mundo era regido por leyes naturales, eternas e inmutables, que postulaban que bajo circunstancias idénticas se obtenían idénticos resultados. Si bien es cierto que en este paradigma se trata lo individual y múltiple, no se los aborda al mismo tiempo, se lo hace de una manera unidimensional y reduccionista, separando lo que está unido (disyunción) y reuniendo lo diverso (reducción).

La noción de pensamiento complejo refiere a la capacidad de interconectar distintas dimensiones de lo real. Ante la emergencia de hechos u objetos multidimensionales, interactivos y con componentes aleatorios o azarosos, el sujeto se ve obligado a de- 
sarrollar una estrategia de pensamiento que no sea reductiva ni totalizante, sino reflexiva. MORIN denominó a dicha capacidad pensamiento complejo. La idea de pensamiento complejo promueve un enfoque transdisciplinario y holístico, aunque sin abandonar la noción de las partes constituyentes del todo. La sistémica, la cibernética y las teorías de la información aportan sustento al pensamiento complejo.

Puede decirse que el pensamiento complejo se basa en tres principios fundamentales: la dialogía (la coherencia del sistema aparece con la paradoja), la recursividad (la capacidad de la retroacción de modificar el sistema) y la hologramía (la parte en el todo y el todo en la parte).

El pensamiento complejo, por lo tanto, es una estrategia o forma del pensamiento que tiene una intención globalizadora o abarcativa de los fenómenos, pero que, a la vez, puede reconocer la especificidad de las partes. La clave pasa por la reorganización de los conocimientos a través de la aplicación de los principios mencionados.

Si el currículo tradicional sienta sus bases en el paradigma de la fragmentación, tanto en sus marcos teórico-conceptuales, en su pedagogía y en su didáctica, es fácil suponer que un currículo que apunte a fomentar una formación más integral debe rediseñar sus componentes, sus relaciones, sus instrumentos desde otros parámetros $y$, desde luego, desde otro marco epistemológico.

\section{HACIA UNA PEDAGOGÍA DE LA COMPLEJIDAD}

La organización curricular de la carrera sigue un modelo clásico, organizada por materias, lo que es lo mismo decir que el objeto de estudio, la arquitectura, es despanzurrada en subcampos disciplinares, en asignaturas ordenadas según una taxo- nomía mecanicista y en compartimentos estancos. Cómo puede explicarse que en el primer año de la carrera se estudie sistema de fundaciones sino fuera porque se cree que debe organizarse el contenido de la asignatura según el orden de materialización en la obra.

Cómo se puede fundamentar científicamente que también en primer año se enseñen conceptos de ciencias básicas totalmente desconectados de los contextos de aplicación. Obviamente que esta manera de organizar el currículo no responde a una malvada y perversa manera de complicar las cosas a los alumnos, sino que es la consecuencia de un paradigma vinculado con el desarrollo exitoso de las ciencias naturales.

Debemos pensar crítica y criteriosamente los fundamentos y las consecuencias de la aplicación de un modelo que, nobleza obliga, se mantiene vigente en la mayor parte del mundo. Cabe preguntarse, entonces, por qué cuestionamos sus bases y sus métodos.

Creo que durante mucho tiempo hubo una aceptación pasiva de las reglas de juego que imponía este enfoque; fue naturalizada la manera de organizar y ejecutar las políticas educativas. Sobre todo fue naturalizada la idea de organizar los contenidos que enseñar en desmedro o ignorando las condiciones del medio y los destinatarios.

Hace algún tiempo podemos constatar la implementación de un modelo diferente en los jardines de infantes. Allí podemos apreciar que los niños desarrollan tareas integradas, vivenciales, con fuerte compromiso emocional, corporal y cognitivo. Tareas estas que se organizan en consonancia con las potencialidades de los sujetos. Cabe señalar que la enseñanza primaria tradicional compensaba la estructuración del conocimiento en parcelas con el rol del maestro único que oficiaba de nexo pedagógico-didáctico entre las materias. 
Pero es en la enseñanza media en donde el sistema muestra descarnadamente sus limitaciones. De parte de los alumnos, imposibilidad de conectar lógica y emocionalmente las diferentes disciplinas. Aburrimiento y tedio en las aulas, a veces indisciplina y conflictos de convivencia.

Los profesores y las intituciones preocupados, pero sin entender por qué no se producen los efectos deseados, a pesar de cumplir con sus tareas y obligaciones. Aparentemente nadie sabe a ciencia cierta dónde radica el problema, será que los alumnos "vienen cada día peor", será culpa de "la disgregación de la familia", etc.

En la universidad la cosa no es muy diferente, aunque el hecho de que la decisión y permanencia sea una opción de los alumnos descomprime el clima de encierro que se percibe en la escuela media.

Tanto la escuela media como la universidad funcionan bien con los buenos alumnos, personas muy adaptadas, que cumplen con las tareas, estudian y tienen buenas notas. Estos alumnos son los que seguramente se graduarán. Sin embargo, hay un problema: una cantidad importante de la matrícula no responde a este estándar, y peor aún, el sistema no puede explicar por qué un sujeto es un buen alumno o por qué no lo es, y mucho menos cómo transformar malos y mediocres alumnos en buenos alumnos. El sistema no puede entender qué ocurre con sus cursantes, porque al sistema nunca le interesó el sujeto alumno. El modelo imperante opera como una suerte de carrera de obstáculos que premia a los que logran sortearlos con cierta habilidad; los que no quieren o no pueden quedan afuera. El abandono de aquellos que no sobreviven a esta prueba es percibido desde el sistema como una falla que se atribuye a las víctimas del sistema.

Lógica consecuencia del capitalismo salvaje o muestra de un darwinismo académico, lo cierto es que estos daños colaterales no son una distorsión del sistema, sino parte de sus rasgos escenciales. Los que quedan afuera siempre es porque o no quieren estudiar o no les gusta trabajar, etc.

Ya en la década del 30 JEAN PIAGET mostró un camino distinto en el campo de la psicología del aprendizaje y la epistemología del conocimiento. En los 60, DAVID AUSUBEL desarrolló la teoría del aprendizaje significativo, y JEROME BRUNER abordó el tema de los aprendizajes de conceptos. En los 80 HOWARD GARDNER propuso su tesis de las inteligencias múltiples. En los últimos treinta años hay innumerables avances en el campo de las neurociencias en sus diferentes vertientes. Todos estos conocimientos, sumados a los planteos de filósofos y pedagogos, nos permiten contar con un importante arsenal de información e instrumentos para comprender cómo es el sistema de aprendizaje del ser humano. Sin embargo, en su gran mayoría nuestras universidades han permanecido impermeables a estos desarrollos científicos, y seguimos enseñando como si esos conocimientos no se hubieran producido. En una conferencia el psicólogo español JOSÉ IGNACIO POZO decía el año pasado que en nuestras aulas enseñamos contenidos del siglo XIX, con profesores del siglo XX y alumnos del siglo XXI.

\section{INCONCLUSIONES}

Recorrimos algunas líneas de reflexión que pretenden confluir en un cauce común que nos ayude a pensar maneras propositivas de cambio, adecuación o adaptación de estructuras académicas que resultaron adecuadas para un mundo que esperaba otras emergencias de los centros de formación profesional y científica. Es importante ver el carácter sistémico del paradigma. No es casual que un mundo racional, simplificado, dividido en partes, estudiado e interpretado en parcelas se resista a aceptar sus limitaciones. Muchas estructuras ins- 
"las disciplinas constituyen un sistema de control en la producción de discurso, fijando sus límites por medio de la acción de una identidad que adopta la forma de una permanente reactivación de las reglas".

titucionales y académicas se reproducen y actúan mientras estas discusiones se desarrollan. Esto me evoca aquella afirmación de FOUCAULT: "las disciplinas constituyen un sistema de control en la producción de discurso, fijando sus límites por medio de la acción de una identidad que adopta la forma de una permanente reactivación de las reglas".

La organización del conocimiento en múltiples disciplinas o materias fragmentadas ha estimulado modelos curriculares e investigativos autorreferentes, convirtiendo un fenómeno convergente en paralelo, e impidiendo cada vez más la integración interdisciplinaria. El paradigma de la educación superior tradicional sigue impulsando esquemas cognitivos disyuntores, que buscan la comprensión y el aprendizaje mediante la separación de las partes, mediante saberes inconexos que no dan espacios para la reflexión, la creación, el disentimiento, la curiosidad y la duda (PEREIRA, 2003). La imbricada red de conexiones entre poderes establecidos, costumbres y tradiciones, todos ellos reforzados y reproducidos por la educación y la investigación, se configuran como condicionantes constrictivas de la vinculación disciplinar en distintos niveles y contextos (ESPINA, 2007).

Un nodo crítico en este escenario será la capacidad de nuestras facultades de arquitectura para organizar las disciplinas ahora aisladas de manera diferente, en un patrón de inteligencia que posibilite el surgimiento de nuevos campos de desarrollo humano, científico y tecnológico. Esto implica la necesidad de propiciar nuevas formas de experimentación cognitiva. Una prospectiva hacia la reintegración de saberes dispersos en distintas disciplinas universitarias, con un énfasis no solo en la acumulación, sino sobre todo en la organización y contextualización cognitiva.

Proponemos enfáticamente la necesidad de una educación abierta a la noción de complejidad con- siderando por lo menos algunas líneas de trabajo, reflexión y debate.

- Rescatar las investigaciones en el campo de ciencias cognitivas y de la educación, que rescatan el carácter irreductible del aprendizaje y su significado, en congruencia con la natural capacidad de las personas y comunidades de percibir y concebir por patrones perceptuales integrados, no en términos de elementos aislados o secuenciales, sino en conjuntos organizados dotados de significado.

- En términos epistemológicos, las disyunciones del conocimiento implican un debilitamiento de la percepción y concepción global, conduciendo al debilitamiento de los sentidos de agenciamiento, pertenencia, responsabilidad y solidaridad social, al desplazar al ámbito de los especialistas y expertos las problemáticas tecnológicas, productivas y sociales de este tiempo.

- La sobrespecialización limita los horizontes de distinciones a escala espacio-tiempo, atentando contra programas de desarrollo a escala humana y la aspiración a una civilización sustentable.

- Las currículas expresan la idea de gestión del conocimiento que tiene una institución, la dimensión normativa que estructura relaciones sociales, campos disciplinares, decisiones de qué se ve y qué no se ve. La decisión de abordar la complejidad implica la intención de establecer un diálogo entre diversos saberes, y esto nos obliga a inventar nuevos instrumentos de relación con los objetos de estudio y con los alumnos.

- Deberíamos propiciar espacios experimentales de didácticas del diseño y observatorios pedagógicos que puedan ir detectando, registrando y evaluando las prácticas, porque justamente de lo que adolecemos es de instrumentos y pautas probadas que puedan orientarnos hacia resultados exitosos. 


\section{BIBLIOGRAFIAA}

ESPINA, M. (2007). Complejidad, transdisciplina y metodología de la investigación social. Utopía y Praxis latinoamericana.

GARCÍA, R. (2007). Sistemas Complejos; Gedisa, Buenos Aires.

MORIN, E. (1988). El Método, El conocimiento del conocimiento; Cátedra. Madrid.

PIAGET, J. (1969). Biología y Conocimiento; Siglo XXI. Buenos Aires.

QUARONI, L. (1977). Proyectar un edificio; Xarait. Madrid.

SIMON, H. (1979). Las Ciencias de lo Artificial; Ate. Barcelona.

VENTURI, R. (1995). Complejidad y Contradicción en la arquitectura. Gili. Barcelona. 